\title{
Analytical Modeling of Transverse Vibrations and Acoustic Pressure Mitigation for Rotating Annular Disks
}

\author{
Hamed Norouzi (iD) ${ }^{1}$ and Davood Younesian (iD) ${ }^{2}$ \\ ${ }^{1}$ Department of Railway Engineering and Transportation Planning, Faculty of Civil Engineering and Transportation, \\ University of Isfahan, Isfahan 81746-73441, Iran \\ ${ }^{2}$ Center of Excellence in Railway Transportation, School of Railway Engineering, Iran University of Science and Technology, \\ Tehran 16846-13114, Iran \\ Correspondence should be addressed to Hamed Norouzi; h.norouzi@eng.ui.ac.ir
}

Received 3 November 2021; Revised 23 December 2021; Accepted 29 December 2021; Published 17 January 2022

Academic Editor: Ji Wang

Copyright ( 2022 Hamed Norouzi and Davood Younesian. This is an open access article distributed under the Creative Commons Attribution License, which permits unrestricted use, distribution, and reproduction in any medium, provided the original work is properly cited.

\begin{abstract}
This paper gives an analytical approach for investigating free and forced transverse vibrations of a clamped-free rotating annular disk and obtaining the acoustic pressure distribution around the spinning disk. In the beginning, a modal analysis based on new analytical methods is carried out to find natural frequencies and mode shapes of the disk. Forced vibration of the disk is then investigated using Galerkin's method. An analytical approach based on Laplace transformation is used to obtain time-dependent coefficients of the transverse response. A passive control strategy is examined for reducing the amplitudes of the transverse vibrations. The properties of the absorbers are examined in order to obtain the best performance. Rayleigh integral method and Durbin's numerical Laplace transform inversion technique are adopted to compute the acoustic pressure around the rotating disk. Finally, a parametric study is performed and the effects of the design parameters as well as rotational conditions on the vibrational responses and the sound pressure of the spinning disk are examined.
\end{abstract}

\section{Introduction}

Many researchers have studied the dynamic behavior of the rotating disks and the acoustic pressure radiated by them because of their wide application in the engineering [1]. The importance of vibrations and acoustic radiation of rotating disks can be found in a different array of practical applications such as circular saws, hard disks, gas turbines, automobile parts, and aerospace structures [2]. In the past decades, some numerical, analytical, and experimental studies have been performed to examine the dynamic analysis of and the acoustic radiation from rotating disks. For example, Qiu et al. [3] examined an active control strategy to control the transverse vibration of a circular disk. The vibration and the noise reduction of an optical disk derived using a vibration absorber was studied by Heo et al. [4], and the required fundamental natural frequency of the absorber was obtained using a finite-element model.
Ciğeroğlu and Özgüven [5] proposed a new model for the vibration analysis of turbine blades with dry friction dampers including both macroslip and microslip models representing dry friction dampers. Koo [6] analyzed the vibration and the critical speeds of polar orthotropic rotating annular disks employing the Rayleigh-Ritz method. Inplane free vibration of circular annular disks was studied by Bashmal et al. [7]. They presented a generalized formulation for the in-plane modal characteristics of circular annular disks under combinations of all possible classical boundary conditions. Hashemi et al. [8] performed vibration analysis of rotating thick plates based on Mindlin plate theory combined with second-order strain-displacement assumptions employing finite-element formulations. Damped vibrations of the double-sided fixed beams placed on a rotational disk were analyzed by Żółkiewski [9]. Younesian et al. [10] analytically studied vibration of a hollow circular plate subjected to a rotating peripheral force adopting 
Galerkin's approach. The influence of shaft's bending on the coupling vibration of a flexible blade-rotor system was analyzed by Li et al. [11]. Elastic stress analysis of a rotating annular disk made of functionally graded material (FGM) with variable thickness was studied by Jalali and Shahriari [12] employing the finite difference method. Bagheri and Jahangiri [13] studied the in-plane free vibration of the functionally graded rotating disks with variable thickness. An accurate solution for the in-plane vibration analysis of rotating circular panels with general edge restraints was proposed by Lyu et al. [14]. The natural frequencies and mode shapes of rotating turbo-machinery components from both rotating and stationary reference frames were experimentally analyzed by Presas et al. [15]. Yang et al. [16] analyzed thermos-elastic coupling vibration and stability of rotating circular plate in friction clutch. Nonlinear vibration analysis of turbine bladed disks with mid-span dampers was studied by Ferhatoglu et al. [17] utilizing Harmonic Balance Method, Alternating Frequency/Time approach, and Newton-Raphson method. Large amplitude vibrations of thinwalled rotating laminated composite cylindrical shell with arbitrary boundary conditions were examined by Li et al. [18] considering the large amplitude vibration of rotating shells with geometric nonlinearity. The coupling vibration characteristics of a rotating disk-beam system with the dovetail interfaces were analyzed by She et al. [19] based on a continuum model. On the other hand, the acoustic radiation from rotating disks or other engineering structures has attracted the attention of many researchers [20]. For instance, the acoustic radiation from out-of-plane modes of an annular disk using thin and thick plate theories was investigated by Lee and Singh [21]. They proposed a semianalytical procedure in which the disk surface velocity is numerically defined by a finite-element model. Reduction of flow-induced vibration and noise of an optical disk drive was performed by Cheng et al. [22]. Maeder et al. [23] studied numerical analysis of sound radiation from rotating disks using a simplified form of the Rayleigh integral known as the lumped parameter model. Recently, the average radiation efficiency of rotating annular plates in the rotating frame was studied analytically by Wang et al. [24] employing Galerkin's method and Rayleigh integral technique. Norouzi and Younesian [25] analyzed the transient and the steady-state sound radiation of nonlinear plates using analytical approaches.

Surveying the literature shows that studies have been addressed the vibrations of the rotating disk do not deal with proposing an analytical model to analyze the flexural vibrations, to mitigate the transverse response, and to reduce the sound radiated from the spinning disk. So, the main contribution of this paper is to represent a closed-form analytical solution in order to cover this gap in the literature. The results of this study can be significant in analyzing the disk brake squeal phenomena or in the study of the noise generated from the railway wheels.

\section{Problem Statement}

2.1. Analytical Model. An isotropic, homogeneous, thin annular disk of inner radius $a$, outer radius $b$, thickness $h$, Young's modulus $E$, mass density $\rho$, and Poisson's ratio $v$ subjected to a constant external load $F$ is shown in Figure 1. The load is applied on the surface of the plate at the coordinate of $\left(r_{p}, \theta_{p}\right)$. The disk has a constant angular speed $\Omega$ about the $z$-axis and is assumed to be rigidly clamped at its inner radius and free at outer radius.

Based on the classical plate theory, the transverse motion of the disk has no significant effect on the in-plane membrane forces of rotation. Moreover, the effects of the gravity and in-plane vibrations are also negligible [26]. So, the governing equation of transverse motion of the disk in cylindrical coordinates $(r, \theta, z)$ can be expressed as [2]

$$
\begin{aligned}
& \frac{\partial^{4} w}{\partial r^{4}}+\frac{2}{r^{2}} \frac{\partial^{4} w}{\partial r^{2} \partial \theta^{2}}+\frac{1}{r^{4}} \frac{\partial^{4} w}{\partial \theta^{4}}+\frac{2}{r} \frac{\partial^{3} w}{\partial r^{3}}-\frac{2}{r^{3}} \frac{\partial^{3} w}{\partial r \partial \theta^{2}}-\frac{1}{r^{2}} \frac{\partial^{2} w}{\partial r^{2}}+\frac{4}{r^{4}} \frac{\partial^{2} w}{\partial \theta^{2}}+\frac{1}{r^{3}} \frac{\partial w}{\partial r} \\
& -\frac{h}{D r}\left(\frac{\partial}{\partial r}\left(r \sigma_{r}^{0} \frac{\partial w}{\partial r}\right)+\frac{\partial}{\partial \theta}\left(\frac{\sigma_{\theta}^{0}}{r} \frac{\partial w}{\partial \theta}\right)\right)+\frac{\rho h}{D}\left(\Omega^{2} \frac{\partial^{2} w}{\partial \theta^{2}}+2 \Omega \frac{\partial^{2} w}{\partial t \partial \theta}+\frac{\partial^{2} w}{\partial t^{2}}\right) \\
= & \frac{F}{D r_{p}} \delta\left(\theta-\theta_{p}\right) \delta\left(r-r_{p}\right),
\end{aligned}
$$

where $w=w(x, y, t)$ is the transverse deflection, $c$ is the viscous damping, and $D=E h^{3} / 12\left(1-v^{2}\right)$ is the rigidity of the disk. Moreover, $\sigma_{r}^{0}$ and $\sigma_{\theta}^{0}$ are the in-plane membrane stresses due to the centrifugal effect. $\delta($.$) also represents$ Dirac's delta function. The boundary conditions for the clamped-free disk are 


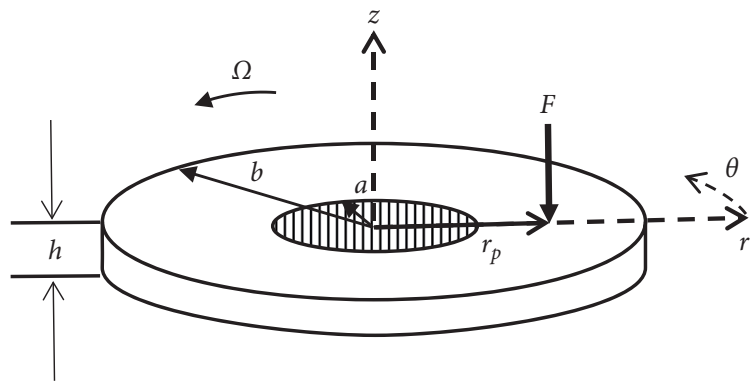

Figure 1: An isotropic, homogeneous, thin annular disk with the rotational speed of $\Omega$.

$$
\begin{aligned}
\left.w\right|_{r=a} & =0 \\
\left.\frac{\partial w}{\partial r}\right|_{r=a} & =0 \\
\left.M_{r}\right|_{r=b} & =0 \Rightarrow D\left(\frac{\partial^{2} w}{\partial r^{2}}+v\left(\frac{1}{r} \frac{\partial w}{\partial r}+\frac{1}{r^{2}} \frac{\partial^{2} w}{\partial \theta^{2}}\right)\right)=0(\text { at } r=b) \\
Q_{r}+\frac{1}{r} \frac{\partial M_{r \theta}}{\partial \theta} & =0 \Rightarrow D\left(\frac{\partial^{3} w}{\partial r^{3}}+\frac{1}{r} \frac{\partial^{2} w}{\partial r^{2}}+\frac{1}{r^{2}} \frac{\partial^{2}}{\partial \theta^{2}}\left(\frac{\partial w}{\partial r}-\frac{w}{r}\right)-\frac{1}{r^{2}}\left(\frac{\partial w}{\partial r}+\frac{1}{r} \frac{\partial^{2} w}{\partial \theta^{2}}\right)+\frac{1-v}{r^{2}}\left(-\frac{1}{r} \frac{\partial^{2} w}{\partial \theta^{2}}+\frac{\partial^{3} w}{\partial r \partial \theta^{2}}\right)\right)=0(\text { at } r=b),
\end{aligned}
$$

in which $Q$ and $M$ are the shear force and the bending moment, respectively. One can employ Galerkin's expansion method and assume $w(r, \theta, t)$ as a periodic function of period $2 \pi$ and write the response of equation (1) as

$w(r, \theta, t)=\sum_{n=0}^{\infty} \sum_{m=0}^{\infty}\left(A_{m n}(t) \cos n \theta+B_{m n}(t) \sin n \theta\right) W_{m}(r)$, where $A_{m n}(t)$ and $B_{m n}(t)$ are time-dependent coefficients and $W_{m}(r)$ is mode shapes of the disk obtained from free vibration analysis.

2.2. Free Vibration Analysis. One can let $F=0$ in equation (1) in order to study the free vibration of the annular disk shown in Figure 1. So, the governing equation can be shown as

$$
\begin{aligned}
& \frac{\partial^{4} w}{\partial r^{4}}+\frac{2}{r^{2}} \frac{\partial^{4} w}{\partial r^{2} \partial \theta^{2}}+\frac{1}{r^{4}} \frac{\partial^{4} w}{\partial \theta^{4}}+\frac{2}{r} \frac{\partial^{3} w}{\partial r^{3}}-\frac{2}{r^{3}} \frac{\partial^{3} w}{\partial r \partial \theta^{2}}-\frac{1}{r^{2}} \frac{\partial^{2} w}{\partial r^{2}}+\frac{4}{r^{4}} \frac{\partial^{2} w}{\partial \theta^{2}}+\frac{1}{r^{3}} \frac{\partial w}{\partial r} \\
& -\frac{h}{D r}\left(\frac{\partial}{\partial r}\left(r \sigma_{r}{ }^{0} \frac{\partial w}{\partial r}\right)+\frac{\partial}{\partial \theta}\left(\frac{\sigma_{\theta}^{0}}{r} \frac{\partial w}{\partial \theta}\right)\right)+\frac{\rho h}{D}\left(\Omega^{2} \frac{\partial^{2} w}{\partial \theta^{2}}+2 \Omega \frac{\partial^{2} w}{\partial t \partial \theta}+\frac{\partial^{2} w}{\partial t^{2}}\right)=0 .
\end{aligned}
$$

Using separation of variables technique (SV) and considering $w$ as a harmonic function, one may suppose the response of equation (4) as

$$
w=\sum_{n=0}^{\infty} \sum_{m=0}^{\infty} w_{m n}=\sum_{n=0}^{\infty} \sum_{m=0}^{\infty} \phi_{m n}(r, \theta) e^{i \omega_{m n} t}
$$

where $\omega_{m n}$ and $\phi_{m n}(r, \theta)$ are the natural frequencies and the corresponding mode shapes, respectively, and $m$ represents the circular mode number and $n$ describes the diametric mode number. Additionally, using periodic property of the response, $\phi_{m n}(r, \theta)$ can also be expressed as

$$
\phi_{m n}(r, \theta)=W_{m}(r) \cos n \theta .
$$

By substituting equation (6) into (5) and the result in equation (3), an ordinary differential equation describing $W(r)$ is obtained as 


$$
\begin{aligned}
& \sum_{n=0}^{\infty} \sum_{m=0}^{\infty}\left(\frac{\mathrm{d}^{4}}{\mathrm{~d} r^{4}}+\frac{2}{r} \frac{\mathrm{d}^{3}}{\mathrm{~d} r^{3}}-\left(2 \frac{n^{2}}{r^{2}}+\frac{1}{r^{2}}+\frac{h}{D} \sigma_{r}^{0}\right) \frac{d^{2}}{d r^{2}}+\left(2 \frac{n^{2}}{r^{3}}+\frac{1}{r^{3}}-\frac{h}{D} \frac{\sigma_{r}^{0}}{r}-\frac{h}{D} \frac{\mathrm{d} \sigma_{r}^{0}}{\mathrm{~d} r}\right) \frac{\mathrm{d}}{\mathrm{d} r}\right. \\
+ & \left.\left(\frac{n^{4}}{r^{4}}-\frac{4 n^{2}}{r^{4}}-\frac{\rho h \Omega^{2}}{D} n^{2}+\frac{h}{D} \sigma_{\theta}^{0} \frac{n^{2}}{r^{2}}\right)\right) \times W_{m}(r) \cos n \theta e^{i \omega_{m n} t}-\frac{2 i \omega_{m n} n \rho h \Omega}{D} W_{m}(r) \sin n \theta e^{i \omega_{m n} t} \\
= & \sum_{n=0}^{\infty} \sum_{m=0}^{\infty} \frac{\rho h}{D} \omega_{m n}{ }^{2} W_{m}(r) \cos n \theta e^{i \omega_{m n} t} m=0,1,2, \ldots, \widehat{M} \quad n=0,1,2, \ldots, \widehat{N} .
\end{aligned}
$$

The corresponding boundary conditions can be derived as follows:

$$
\begin{aligned}
\left.w\right|_{r=a}= & \left.\Rightarrow W_{m}(r)\right|_{r=a}=0, \\
\left.\frac{\partial w}{\partial r}\right|_{r=a}= & \left.0 \Rightarrow \frac{\mathrm{d} W_{m}(r)}{\mathrm{d} r}\right|_{r=a}=0, \\
& \left\{\frac{\mathrm{d}^{2} W_{m}(r)}{\mathrm{d} r^{2}}+v\left(\frac{1}{r} \frac{\mathrm{d} W_{m}(r)}{\mathrm{d} r}-\frac{n^{2}}{r^{2}} W_{m}(r)\right)\right\}_{r=b}=0, \\
& \left\{\frac{\mathrm{d}^{3} W_{m}(r)}{\mathrm{d} r^{3}}+\frac{1}{r} \frac{\mathrm{d}^{2} W_{m}(r)}{\mathrm{d} r^{2}}-\frac{1}{r^{2}}\left((2+v) n^{2}+1\right) \frac{\mathrm{d} W_{m}(r)}{\mathrm{d} r}+\frac{(3+v)}{r^{3}} n^{2} W_{m}(r)\right\}_{r=b}=0 .
\end{aligned}
$$

For solving equation (7) with boundary conditions (8), one may multiply both sides of equation (7) by $r W_{m}(r) \cos n \theta$ and perform integral from $a$ to $b$ for $r$ and 0 to $2 \pi$ for $\theta$ and then use the orthogonality property of the mode shapes of the disk $[1,20]$ and obtain

$$
\begin{aligned}
& \left(\frac{\mathrm{d}^{4}}{\mathrm{~d} r^{4}}+\frac{2}{r} \frac{\mathrm{d}^{3}}{\mathrm{~d} r^{3}}-\left(2 \frac{n^{2}}{r^{2}}+\frac{1}{r^{2}}+\frac{h}{D} \sigma_{r}^{0}\right) \frac{\mathrm{d}^{2}}{\mathrm{~d} r^{2}}+\left(2 \frac{n^{2}}{r^{3}}+\frac{1}{r^{3}}-\frac{h}{D} \frac{\sigma_{r}^{0}}{r}-\frac{h}{D} \frac{\mathrm{d} \sigma_{r}^{0}}{\mathrm{~d} r}\right) \frac{\mathrm{d}}{\mathrm{d} r}\right. \\
& \left.+\left(\frac{n^{4}}{r^{4}}-\frac{4 n^{2}}{r^{4}}-\frac{\rho h \Omega^{2}}{D} n^{2}+\frac{h}{D} \sigma_{\theta}^{0} \frac{n^{2}}{r^{2}}\right)\right) W_{m}(r) \\
= & \frac{\rho h}{D} \omega_{m n}{ }^{2} W_{m}(r) m=0,1,2, \ldots, \widehat{M} \quad n=0,1,2, \ldots, \widehat{N} .
\end{aligned}
$$

Here, $\widehat{M}$ and $\widehat{N}$ are arbitrary parameters that show the upper bound for the mode numbers and they are both specified. Equation (9) forms an eigenvalue-eigenfunction problem. The solution of equation (9) can be written as follows [27]:

$$
W_{m}(r)=\sum_{j=0}^{P} c_{j, m} r^{j}+R_{P, m}(r),
$$

where $c_{j}$ and $R_{P}(j=1,2, \ldots, P)$ are unknown linear independent coefficients and the rest, respectively, and $P$ is a certain positive integer which is chosen large enough such that the rest has a negligible error. Therefore, neglecting $R_{P}$ in equation (9), the application of conditions (8) leads to yield two linear equations of $P+1$ unknown coefficients of $c_{0}, c_{1}, c_{2}, \ldots, c_{P}$ as follows:

$$
\begin{aligned}
& \sum_{j=0}^{P}\left(h_{1 j}-\lambda k_{1 j}\right) c_{j}=0, \\
& \sum_{j=0}^{P}\left(h_{2 j}-\lambda k_{2 j}\right) c_{j}=0,
\end{aligned}
$$


with

$$
\begin{aligned}
h_{1 j}= & a^{j}, \\
k_{1 j}= & 0 \\
& (j=0,1,2, \ldots, P), \\
h_{2 j}= & j a^{j-1}, \\
k_{2 j}= & 0 \\
& (j=0,1,2, \ldots, P) .
\end{aligned}
$$

On the other hand, using conditions (8), one has

$$
\begin{aligned}
& \sum_{j=0}^{P}\left(h_{3 j}-\lambda k_{3 j}\right) c_{j}=0 \\
& \sum_{j=0}^{P}\left(h_{4 j}-\lambda k_{4 j}\right) c_{j}=0
\end{aligned}
$$

with

$$
\begin{aligned}
h_{3 j}= & \sum_{j=0}^{P} j(j-1) b^{j-2}+v\left(\frac{1}{b} \sum_{j=0}^{P} j b^{j-1}-\frac{n^{2}}{b^{2}} \sum_{j=0}^{P} b^{j}\right), \\
k_{3 j}= & 0 \quad(j=0,1,2, \ldots, P), \\
h_{2 j}= & \sum_{j=0}^{P} j(j-1)(j-2) b^{j-3}+\frac{1}{b} \sum_{j=0}^{P} j b^{j-1} \\
& -\frac{1}{b^{2}}\left((2+v) n^{2}+1\right) \sum_{j=0}^{P} j b^{j-1}+\frac{(3+v)}{b^{3}} n^{2} \sum_{j=0}^{P} b^{j}, \\
k_{4 j}= & 0 \quad(j=0,1,2, \ldots, P) .
\end{aligned}
$$

Equations (11) through (14) give four expressions about the unknowns of $c_{i}$. But, according to equation (10), without considering $\mathrm{R}_{P, m}$, one needs obtaining $P+1$ coefficients. So, another $\mathrm{P}-3$ independent equation should be produced. Inserting polynomial equation (10) into (9) and multiplying both sides of the result by $r^{i}(i=4,5,6, \ldots, P)$ and then integrating with respect to $r$ between $a$ and $b$ lead to

$$
\sum_{j=0}^{P}\left(h_{i j}-\lambda k_{i j}\right) c_{j}=0 \quad(i=4,5,6, \ldots P),
$$

with

$$
\begin{aligned}
& h_{i j}=\int_{a}^{b}\left(\mathfrak{J}\left\{\sum_{j=0}^{P} r^{j+i}\right\}\right) r d r, \\
& k_{i j}=\frac{b^{j+i}-a^{j+i}}{j+i}(i=4,5,6, \ldots P),
\end{aligned}
$$

in which the operator $\mathfrak{\Im}$ is

$$
\begin{aligned}
\mathfrak{\Im}: \frac{\mathrm{d}^{4}}{\mathrm{~d} r^{4}} & +\frac{2}{r} \frac{\mathrm{d}^{3}}{\mathrm{~d} r^{3}}-\left(2 \frac{n^{2}}{r^{2}}+\frac{1}{r^{2}}+\frac{h}{D} \sigma_{r}^{0}\right) \frac{\mathrm{d}^{2}}{\mathrm{~d} r^{2}}+\left(2 \frac{n^{2}}{r^{3}}+\frac{1}{r^{3}}-\frac{h}{D} \frac{\sigma_{r}^{0}}{r}-\frac{h}{D} \frac{\mathrm{d} \sigma_{r}^{0}}{\mathrm{~d} r}\right) \frac{\mathrm{d}}{\mathrm{d} r} \\
& +\left(\frac{n^{4}}{r^{4}}-\frac{4 n^{2}}{r^{4}}-\frac{\rho h \Omega^{2}}{D} n^{2}+\frac{h}{D} \sigma_{\theta}^{0} \frac{n^{2}}{r^{2}}\right) .
\end{aligned}
$$

Therefore, equations (11), (13), and (15) form a system of $P+1$ linear algebraic equations for $P+1$ unknown coefficients $c_{i}(i=0,1, \ldots, P)$, which can be rewritten in a compact form as

$$
(H-\lambda K)_{(P+1) \times(P+1)} \times\left(c_{0} c_{1} c_{2} \ldots c_{P}\right)^{T}=0,
$$

where

$$
\begin{aligned}
H & =h_{j i(P+1) \times(P+1)}, \\
K & =k_{j i(P+1) \times(P+1)}, \\
\lambda & =\frac{\rho h}{D} \omega^{2} .
\end{aligned}
$$

To obtain a nontrivial solution of the system of equation (19), the determinant of the coefficient matrix has to vanish. Then, one gets a characteristic equation in eigenvalues $\lambda$ as 


$$
\operatorname{det}(H-\lambda K)=0 .
$$

Solving equation (20), one can obtain the natural frequencies of the disk. After finding the natural frequencies, the coefficient matrix $c$ is obtained from equation (18) and, finally, equation (10) gives the mode shapes.
2.3. Forced Vibration Analysis. Recalling equations (1) and (3), one can replace equation (3) in (1) and multiply both sides of the result first by $r W_{m}(r) \cos n \theta$ and then by $r W_{m}(r) \sin n \theta$ and finally perform integration from 0 to $2 \pi$ in $\theta$ and from $a$ to $b$ in $r$ to give a system of coupled ordinary differential equations as follows:

$$
\begin{aligned}
& \frac{\mathrm{d}^{2} A_{m n}(t)}{\mathrm{d} t^{2}}+2 n \Omega \frac{\mathrm{d} B_{m n}(t)}{\mathrm{d} t}+\left(\omega_{m n}^{2}-n^{2} \Omega^{2}\right) A_{m n}(t)+F W_{m}\left(r_{p}\right)=0, \\
& \frac{\mathrm{d}^{2} B_{m n}(t)}{\mathrm{d} t^{2}}-2 n \Omega \frac{\mathrm{d} A_{m n}(t)}{\mathrm{d} t}+\left(\omega_{m n}^{2}-n^{2} \Omega^{2}\right) B_{m n}(t)=0 m=0,1,2, \ldots, \widehat{M} \quad n=0,1,2, \ldots, \widehat{N} .
\end{aligned}
$$

Equation (21) can be solved employing Laplace transform technique. By taking Laplace transform from system of equation (21), one has

$$
\begin{aligned}
& s^{2} \widehat{A}_{m n}(s)+\alpha_{m n} \widehat{A}_{m n}(s)+\beta_{n} s \widehat{B}_{m n}(s)+\frac{1}{s} F W_{m}\left(r_{p}\right)=0, \\
& s^{2} \widehat{B}_{m n}(s)+\alpha_{m n} \widehat{B}_{m n}(s)-\beta_{n} s \widehat{A}_{m n}(s)=0 m=0,1,2, \ldots, \widehat{M} \quad n=0,1,2, \ldots, \widehat{N},
\end{aligned}
$$

in which

$$
\begin{aligned}
& \widehat{A}_{m n}(s)=\int_{0}^{\infty} A_{m n}(t) e^{-s t} d t, \\
& \widehat{B}_{m n}(s)=\int_{0}^{\infty} B_{m n}(t) e^{-s t} d t .
\end{aligned}
$$

$$
\begin{aligned}
& \widehat{A}_{m n}(s)=\frac{-\left(s^{2}+\alpha_{m n}\right)}{\left(s^{2}+\alpha_{m n}\right)^{2}+s^{2} \beta_{n}^{2}} \frac{1}{s} F W_{m}\left(r_{p}\right), \\
& \widehat{B}_{m n}(s)=\frac{-s \beta_{n}}{\left(s^{2}+\alpha_{m n}\right)^{2}+s^{2} \beta_{n}^{2}} \frac{1}{s} F W_{m}\left(r_{p}\right) .
\end{aligned}
$$

Solving the algebraic equation (22) leads to obtain $\widehat{A}_{m n}(s)$ and $\widehat{B}_{m n}(s)$ as

$$
\begin{aligned}
& A_{m n}(t)=\frac{F W_{m}\left(r_{p}\right)}{2\left(r_{m n, 1}^{2}-r_{m n, 2}^{2}\right)}\left\{\left(\frac{r_{m n, 1}^{2}+\alpha_{m n}}{r_{m n, 1}^{2}}\right)\left(-e^{r_{m n, 1} t}-e^{-r_{m n, 1} t}\right)+\left(\frac{r_{m n, 2}^{2}+\alpha_{m n}}{r_{m n, 2}^{2}}\right)\left(e^{r_{m n, 2} t}+e^{-r_{m n, 2} t}\right)-\frac{2 \alpha_{m n}\left(r_{m n, 1}^{2}-r_{m n, 2}^{2}\right)}{r_{m n, 1}^{2} r_{m n, 2}^{2}}\right\}, \\
& B_{m n}(t)=\frac{F W_{m}\left(r_{p}\right) \beta_{n}}{2\left(r_{m n, 1}^{2}-r_{m n, 2}^{2}\right)}\left\{\frac{e^{r_{m n, 1} t}-e^{-r_{m n, 1} t}}{r_{m n, 1}}-\frac{e^{r_{m n, 2} t}-e^{-r_{m n, 2} t}}{r_{m n, 2}}\right\},
\end{aligned}
$$

The Laplace transform inversion scheme is now employed to obtain $A_{m n}(t)$ and $B_{m n}(t)$ as where

$$
\begin{aligned}
& r_{m n, 1}=\frac{-i \beta_{n}+i \sqrt{\beta_{n}^{2}+4 \alpha_{m n}}}{2} \\
& r_{m n, 2}=\frac{-i \beta_{n}-i \sqrt{\beta_{n}^{2}+4 \alpha_{m n}}}{2} .
\end{aligned}
$$

And, $i=\sqrt{-1}$ is the unit imaginary number. Replacing equation (25) in conjunction with $W_{m}(r)$ acquired in the previous section into equation (3), the transverse vibrational response of the disk is determined.

2.4. Vibration Absorption. In this section, the reduction in the vibrational deflection of the annular rotating disk is proposed by adding TMDs to the surface of the disk 
properly. Each TMD has the mass of $m_{q}$, the stiffness of $k_{q}$, and the viscous damping of $c_{q}(q=1,2,3, \ldots, Q)$, respectively, and is placed on the surface of the rotating disk at the coordinate of $\left(r_{q}, \theta_{q}\right)$ as shown in Figure 2.
Based on equation (1), the equation of transverse motion of the disk-TMDs system can be expressed as

$$
\begin{aligned}
& \frac{\partial^{4} w}{\partial r^{4}}+\frac{2}{r^{2}} \frac{\partial^{4} w}{\partial r^{2} \partial \theta^{2}}+\frac{1}{r^{4}} \frac{\partial^{4} w}{\partial \theta^{4}}+\frac{2}{r} \frac{\partial^{3} w}{\partial r^{3}}-\frac{2}{r^{3}} \frac{\partial^{3} w}{\partial r \partial \theta^{2}}-\frac{1}{r^{2}} \frac{\partial^{2} w}{\partial r^{2}}+\frac{4}{r^{4}} \frac{\partial^{2} w}{\partial \theta^{2}}+\frac{1}{r^{3}} \frac{\partial w}{\partial r} \\
& -\frac{h}{D r}\left(\frac{\partial}{\partial r}\left(r \sigma_{r}^{0} \frac{\partial w}{\partial r}\right)+\frac{\partial}{\partial \theta}\left(\frac{\sigma_{\theta}^{0}}{r} \frac{\partial w}{\partial \theta}\right)\right)+\frac{\rho h}{D}\left(\Omega^{2} \frac{\partial^{2} w}{\partial \theta^{2}}+2 \Omega \frac{\partial^{2} w}{\partial t \partial \theta}+\frac{\partial^{2} w}{\partial t^{2}}\right) \\
& =\frac{F}{D r_{p}} \delta\left(\theta-\theta_{p}\right) \delta\left(r-r_{p}\right)+\sum_{q=1}^{Q} \Gamma_{q}(t) \frac{\delta\left(r-r_{q}\right) \delta\left(\theta-\theta_{q}\right)}{r},
\end{aligned}
$$

where $\Gamma_{q}(t)$ is the force acting from $q^{t h}$ TMD on the surface of the disk. The equation of the motion of the $q^{\text {th }}$ TMD can also be shown as follows:

$$
\begin{array}{r}
\frac{\mathrm{d}^{2} u_{q}}{\mathrm{~d} t^{2}}+2 \zeta_{q} \omega_{q}\left(\frac{\mathrm{d} u_{q}}{\mathrm{~d} t}-\frac{\mathrm{d} w_{q}}{\mathrm{~d} t}\right)+\omega_{q}^{2}\left(u_{q}-w_{q}\right)=0, \\
\Gamma_{q}(t)=m_{q}\left\{2 \zeta_{q} \omega_{q}\left(\frac{\mathrm{d} u_{q}}{\mathrm{~d} t}-\frac{\mathrm{d} w_{q}}{\mathrm{~d} t}\right)+\omega_{q}^{2}\left(u_{q}-w_{q}\right)\right\},
\end{array}
$$

in which $u_{q}=u_{q}(t)$ is the displacement, $\omega_{q}=\sqrt{k_{q} / m_{q}}$ is the natural frequency, and $\zeta_{q}=c_{q} / 2 \sqrt{m_{q} k_{q}}$ is the damping ratio of the dynamic absorber, respectively. Moreover, $w_{g}$ is the deflection of the disk at the point where $q^{\text {th }}$ TMD is connected. Putting $Q=1$ and considering an absorber of mass $m_{a}$, the natural frequency of $\omega_{a}$, and the damping ratio of $\zeta_{a}$ connected to the point of $\left(r_{a}, \theta_{a}\right)$ on the surface of the disk, one can write

$$
\begin{aligned}
& \frac{\partial^{4} w}{\partial r^{4}}+\frac{2}{r^{2}} \frac{\partial^{4} w}{\partial r^{2} \partial \theta^{2}}+\frac{1}{r^{4}} \frac{\partial^{4} w}{\partial \theta^{4}}+\frac{2}{r} \frac{\partial^{3} w}{\partial r^{3}}-\frac{2}{r^{3}} \frac{\partial^{3} w}{\partial r \partial \theta^{2}}-\frac{1}{r^{2}} \frac{\partial^{2} w}{\partial r^{2}}+\frac{4}{r^{4}} \frac{\partial^{2} w}{\partial \theta^{2}}+\frac{1}{r^{3}} \frac{\partial w}{\partial r} \\
& -\frac{h}{D r}\left(\frac{\partial}{\partial r}\left(r \sigma_{r}^{0} \frac{\partial w}{\partial r}\right)+\frac{\partial}{\partial \theta}\left(\frac{\sigma_{\theta}^{0}}{r} \frac{\partial w}{\partial \theta}\right)\right)+\frac{\rho h}{D}\left(\Omega^{2} \frac{\partial^{2} w}{\partial \theta^{2}}+2 \Omega \frac{\partial^{2} w}{\partial t \partial \theta}+\frac{\partial^{2} w}{\partial t^{2}}\right) \\
= & \frac{F}{D r_{p}} \delta\left(\theta-\theta_{p}\right) \delta\left(r-r_{p}\right)+\Gamma_{a}(t) \frac{\delta\left(r-r_{a}\right) \delta\left(\theta-\theta_{a}\right)}{r},
\end{aligned}
$$

where $\Gamma_{a}(t)$ is the contact force created between the dynamic absorber and the disk. Equation (29) should be solved considering equation (28). Utilizing Galerkin's approach, the solution can be expressed as

$w(r, \theta, t)=\sum_{n=0}^{\infty} \sum_{m=0}^{\infty}\left(E_{m n}(t) \cos n \theta+F_{m n}(t) \sin n \theta\right) W_{m}(r)$, where $E_{m n}(t)$ and $F_{m n}(t)$ are unknown coefficients. Substituting equation (30) into (29) and multiplying both sides of the result first by $r W_{m}(r) \cos n \theta$ and performing integration from 0 to $2 \pi$ in $\theta$ and from $a$ to $b$ in $r$ and then by $r W_{m}(r) \sin n \theta$ and performing the same integrations constructs a system of coupled ordinary differential equations as follows: 


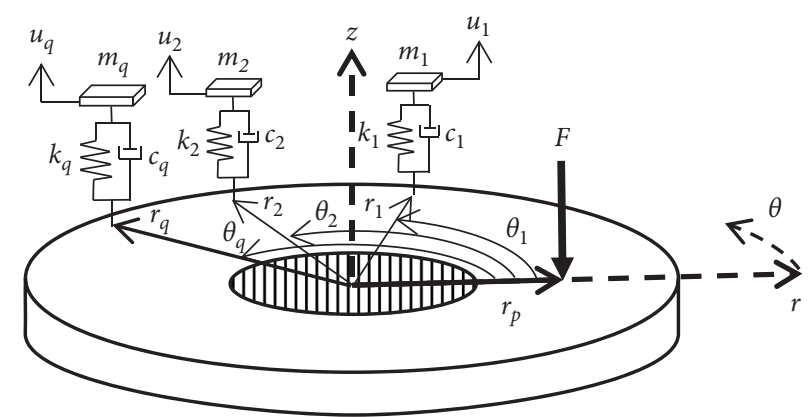

Figure 2: TMDs placed on the surface of the rotating disk.

$$
\begin{aligned}
& \frac{\mathrm{d}^{2} E_{i j}}{\mathrm{~d} t^{2}}+2 j \Omega \frac{\mathrm{d} F_{i j}}{\mathrm{~d} t}+\left(\omega_{i j}^{2}-j^{2} \Omega^{2}\right) E_{i j}+2 \zeta_{i j} \omega_{i j}\left(\frac{\mathrm{d} E_{i j}}{\mathrm{~d} t}+j \Omega F_{i j}\right)+F Y_{i}\left(r_{p}\right)-\Gamma_{a}(t) Y_{i}\left(r_{a}\right) \cos \left(j \theta_{a}\right)=0, \\
& \frac{\mathrm{d}^{2} F_{i j}}{\mathrm{~d} t^{2}}-2 j \Omega \frac{\mathrm{d} E_{i j}}{\mathrm{~d} t}+\left(\omega_{i j}^{2}-j^{2} \Omega^{2}\right) F_{i j}+2 \zeta \omega_{i j}\left(\frac{\mathrm{d} F_{i j}}{\mathrm{~d} t}-j \Omega E_{i j}\right)-\Gamma_{a}(t) Y_{i}\left(r_{a}\right) \sin \left(j \theta_{a}\right)=0,
\end{aligned}
$$

in which $(i=0,1,2, \ldots, \widehat{M}, j=0,1,2, \ldots, \widehat{N})$. Considering equations (29) and (28) and letting $w_{a}(t)=\sum_{n=0}^{\infty}$

$\sum_{m=0}^{\infty}\left(E_{m n}(t) \cos n \theta_{a}+F_{m n}(t) \sin n \theta_{a}\right) W_{m}\left(r_{a}\right)$, one can obtain

$$
\begin{aligned}
& \frac{\mathrm{d}^{2} E_{i j}}{\mathrm{~d} t^{2}}+2 j \Omega \frac{\mathrm{d} F_{i j}}{\mathrm{~d} t}+\left(\omega_{i j}^{2}-j^{2} \Omega^{2}\right) E_{i j}+2 \zeta_{i j} \omega_{i j}\left(\frac{\mathrm{d} E_{i j}}{\mathrm{~d} t}+j \Omega F_{i j}\right)+F Y_{i}\left(r_{p}\right) \\
& -m_{a}\left(2 \zeta_{a} \omega_{a}\left(\frac{\mathrm{d} u_{a}}{\mathrm{~d} t}-\frac{\mathrm{d} E_{i j}}{\mathrm{~d} t}-\frac{\mathrm{d} F_{i j}}{\mathrm{~d} t}\right)+\omega_{a}^{2}\left(u_{a}-E_{i j}-F_{i j}\right)\right) Y_{i}\left(r_{a}\right) \cos \left(j \theta_{a}\right)=0, \\
& \frac{\mathrm{d}^{2} F_{i j}}{\mathrm{~d} t^{2}}-2 j \Omega \frac{\mathrm{d} E_{i j}}{\mathrm{~d} t}+\left(\omega_{i j}^{2}-j^{2} \Omega^{2}\right) F_{i j}+2 \zeta \omega_{i j}\left(\frac{\mathrm{d} F_{i j}}{\mathrm{~d} t}-j \Omega E_{i j}\right) \\
& -m_{a}\left(2 \zeta_{a} \omega_{a}\left(\frac{\mathrm{d} u_{a}}{\mathrm{~d} t}-\frac{\mathrm{d} E_{i j}}{\mathrm{~d} t}-\frac{\mathrm{d} F_{i j}}{\mathrm{~d} t}\right)+\omega_{a}^{2}\left(u_{a}-E_{i j}-F_{i j}\right)\right) Y_{i}\left(r_{a}\right) \sin \left(j \theta_{a}\right)=0, \\
& \frac{\mathrm{d}^{2} u_{a}}{\mathrm{~d} t^{2}}+2 \zeta_{a} \omega_{a}\left(\frac{\mathrm{d} u_{a}}{\mathrm{~d} t}-\frac{\mathrm{d} E_{i j}}{\mathrm{~d} t}-\frac{\mathrm{d} F_{i j}}{\mathrm{~d} t}\right)+\omega_{a}^{2}\left(u_{a}-E_{i j}-F_{i j}\right) .
\end{aligned}
$$

Equation (32) is a set of ordinary differential equations which can be solved using numerical methods. The Runge-Kutta method is employed here in order to solve equation (32) and to determine the parameters of $\operatorname{Eij}(t)$, Fij(t), and ua(t). So, obtaining the parameters of Eij(t), Fij(t), and ua( $\mathrm{t})$, one can obtain the transverse vibrational response of the system of disk-absorber using equation (30).

2.5. Acoustic Radiation. Figure 3 shows the disk of Figure 1 radiating the sound pressure. The sound pressure distribution $P(r, \theta, z, t)$ at an arbitrary point $G$ can be obtained based on Rayleigh integral as follows $[25,28]$ :

$$
P\left(r_{G}, \theta_{G}, z_{G}, t\right)=\frac{\rho_{0}}{2 \pi} \int_{0}^{2 \pi} \int_{a}^{b} \frac{d^{2}}{d t^{2}} w\left(r, \theta, t-\frac{R}{c_{0}}\right) \frac{1}{R} r d r d \theta,
$$

where $d^{2} w / d t^{2}$ denotes the acceleration of the disk, $\rho_{0}$ and $c_{0}$ are the mass density and the sound speed of the acoustic medium, respectively, and $R=\sqrt{z_{G}^{2}+r_{G}^{2}+r^{2}-2 r_{G} r \cos \left(\theta_{G}-\theta\right)}$ is the distance between the observation point $G$ and an element on the surface of the disk at the location of $(r, \theta)$. Laplace transform technique can be employed again to obtain the acoustic pressure of equation (33). By taking Laplace transform from both sides of equation (33), one has 


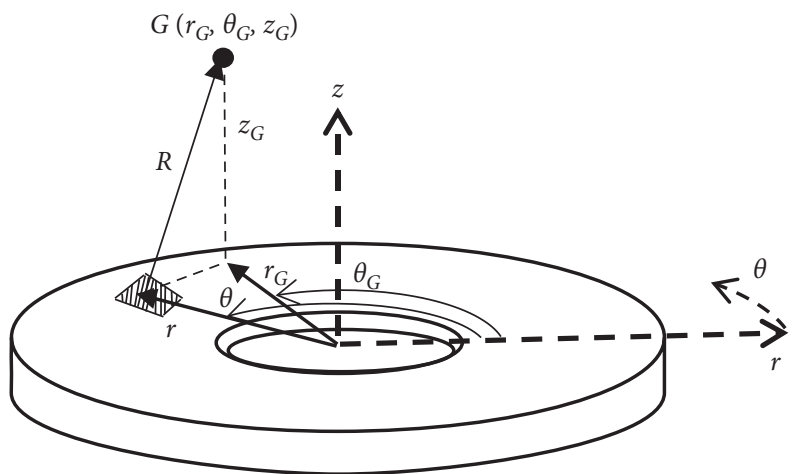

Figure 3: The observation point $(G)$ in which the acoustic pressure should be determined.

$$
\widehat{P}\left(r_{G}, \theta_{G}, z_{G}, s\right)=\frac{\rho_{0} s^{2}}{2 \pi} \int_{0}^{2 \pi} \int_{a}^{b} e^{-R / c_{0} s} w(r, \theta, s) \frac{1}{R} r d r d \theta,
$$

in which $\widehat{P}\left(r_{G}, \theta_{G}, z_{G}, s\right)=\int_{0}^{+\infty} P\left(r_{G}, \theta_{G}, z_{G}, t\right) e^{-s t} d t$. Employing the Laplace transform inversion scheme, one can acquire the acoustic pressure. Durbin's approach is used to obtain Laplace transform inversion of equation (34) as [29]

$$
\begin{aligned}
P(t)= & \frac{2 e^{\eta t}}{\tau} \times\left\{\frac{1}{2} \operatorname{Re}(\widehat{P}(\eta))+\sum_{m=1}^{M}\left[\operatorname{Re}\left(\widehat{P}\left(\eta+i \frac{2 m \pi}{\tau}\right)\right) \cos \left(\frac{2 m \pi}{\tau} t\right)\right.\right. \\
& \left.\left.-\operatorname{Im}\left(\widehat{P}\left(\eta+i \frac{2 m \pi}{\tau}\right)\right) \sin \left(\frac{2 m \pi}{\tau} t\right)\right]\right\},
\end{aligned}
$$

where $\eta$ is an arbitrary real number greater than all the real parts of the singularities of $\widehat{P}(\eta)$ and $\widehat{P}(\eta)$ should be defined in the interval $[0,2 \tau]$. For sufficient accuracy, the suggested value of " $\eta t$ " is given with the appropriate sign by [30]

$$
\eta t=-2 \ln \left\{N_{\tau}\right\}
$$

where $N \tau$ is the number of points in the time signal.

\section{Results and Discussion}

In this section, a parametric study is performed in order to investigate the effects of different parameters on the vibrational response and the acoustic pressure radiated from the spinning annular disk. In Table 1, the natural frequencies of the rotating disk obtained from the present study have been compared with the results from other references. The agreement between the results shows the accuracy of the proposed analytical approach. Table 2 also contains the geometrical properties of the disk and the physical properties of the acoustic medium.

Figures 4(a) through 4(f) show the mode shapes and the natural frequencies of the rotating annular disk for the first six modes. Time-dependent coefficients of $A_{m n}(t)$ and $B_{m n}(t)$ are plotted in Figure 5 against the time. The figures have been plotted using two methods: the first graph in each figure by using equation (25) and the second one employing the numerical Runge-Kutta approach. There are excellent agreements between the results.
Figures $6(\mathrm{a})-6(\mathrm{~d})$ compare vibrational response of the disk observed at the point with coordinate of $(0.35, \pi / 4)$ for $\Omega=5 \pi \mathrm{rad} / \mathrm{s}$ and for different loading amplitudes. It can be found from these figures that increasing the amplitude of the external load causes the dynamical response to be more regular. For fully forced vibration of the disk (Figure 6(d)), one can see that the period of the response $(0.4 \mathrm{sec})$ is the same as the rotational period.

The effect of the dynamic absorber attaching on the surface of the disk has been illustrated in Figures $7(a)-7(d)$. The figures have been plotted with and without the absorber existence and for different frequencies of the TMD. It can be seen in Figure 7 that the efficiency of the TMD is variable in different natural frequencies. For finding the best natural frequency of the dynamic absorber, the root mean square (RMS) of the response has been shown against the natural frequency of the TMD in Figure 8. It is clear from Figure 8 that the efficiency of the absorber has the maximum value in a certain natural frequency (about $2100 \mathrm{~Hz}$ in this study). Moreover, the frequencies in which the deflection is not restrained well (about $1700 \mathrm{~Hz}$ and $2800 \mathrm{~Hz}$ ), actually, are the natural frequencies of the system of disk-absorber. Figure 8 also shows that the performance of the TMD is impaired by increasing its natural frequency after a certain frequency (about $4 \mathrm{KHz}$ in our study).

Figure 9 displays RMS of the transverse response versus the natural frequency of the TMD which is located in the different places on the surface of the disk. It is found from Figure 9 that the absorber location plays an important role in the performance of the vibration absorber. Figure 9 also shows that for the natural frequencies of the TMD after a certain frequency (about $3000 \mathrm{~Hz}$ in this study), the location of the TMD has no significant effect on the disk response. Moreover, at any location where the absorber placed on, the TMD has a maximum and a minimum effectiveness.

As it is shown in Figure 8, one can choose the best TMD from the natural frequency point of view. On the other hand, Figure 9 gives the best location on the surface of the disk in which the attached TMD has the best efficiency $(407.5 \mathrm{~mm}$ from the center of the disk in this study). In fact, considering Figures 8 and 9, one can choose the best characters of TMD with the optimum performance. Figure 10 shows the time history of the transverse response of the disk before and after using the best dynamic absorber. From Figure 10, one can 
TABLe 1: The natural frequencies $(\mathrm{Hz})$ of the rotating disk compared with the results of Ref. [2].

\begin{tabular}{|c|c|c|c|c|c|}
\hline Mode number & Ref. [2] & Present study & Mode number & Ref. [2] & Present study \\
\hline$(1,1)$ & 478 & 480 & $(2,1)$ & 3100 & 3106 \\
\hline$(1,2)$ & 484 & 485 & $(2,2)$ & 3118 & 3119 \\
\hline$(1,3)$ & 506 & 506 & $(2,3)$ & 3175 & 3175 \\
\hline$(1,4)$ & 550 & 553 & $(2,4)$ & 3268 & 3269 \\
\hline
\end{tabular}

TABle 2: The properties of the studied disk and the acoustic medium.

\begin{tabular}{lc}
\hline Item & Value \\
\hline Disk outer radius $(b)$ & $0.5 \mathrm{~m}$ \\
Disk inner radius $(a)$ & $0.2 \mathrm{~m}$ \\
Disk thickness $(h)$ & $4 \mathrm{~mm}$ \\
Disk Young's modulus $(E)$ & $210 \mathrm{GPa}$ \\
Disk Poisson's ratio $(v)$ & 0.3 \\
Disk mass density $(\rho)$ & $7800 \mathrm{Kg} / \mathrm{m}^{3}$ \\
Acoustic medium mass density $\left(\rho_{0}\right)$ & $1.14 \mathrm{Kg} / \mathrm{m}^{3}$ \\
Sound speed $\left(c_{0}\right)$ & $340 \mathrm{~m} / \mathrm{s}$ \\
\hline
\end{tabular}

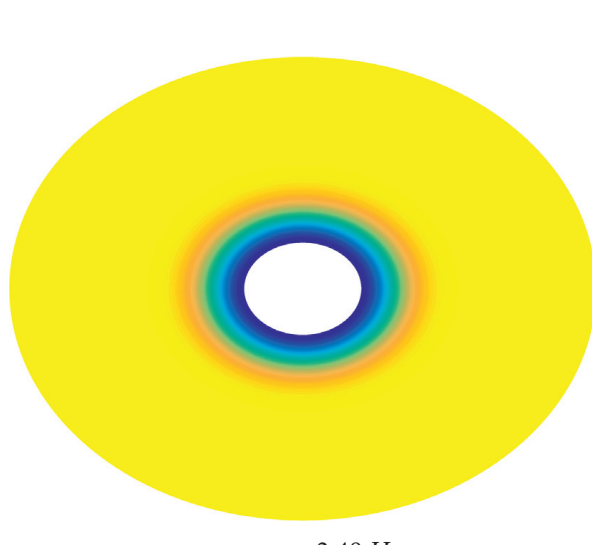

$\omega_{0,0}=2.48 \mathrm{~Hz}$

(a)

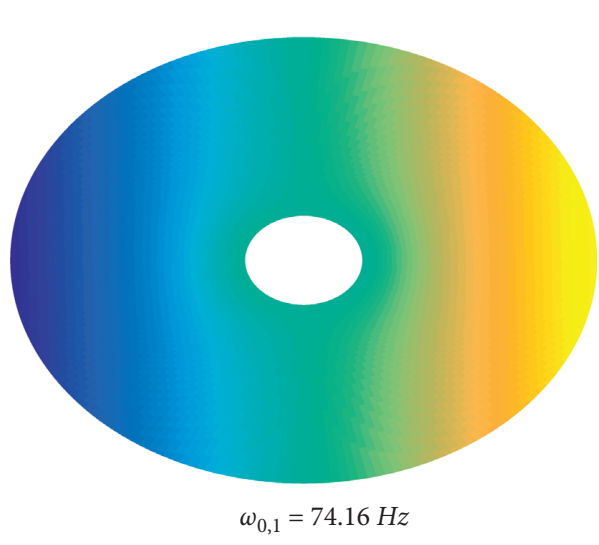

(c)
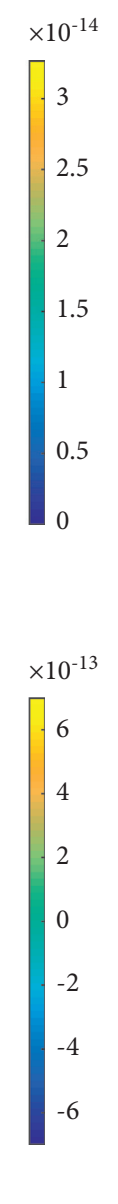

Figure 4: Continued.

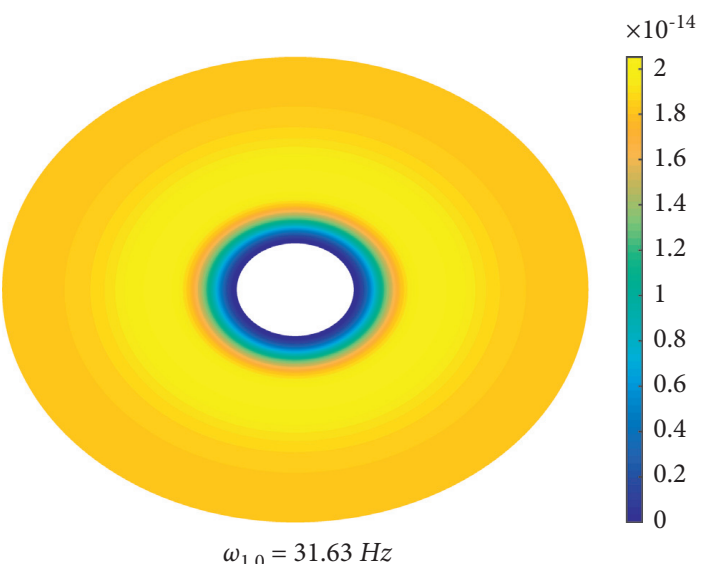

(b)

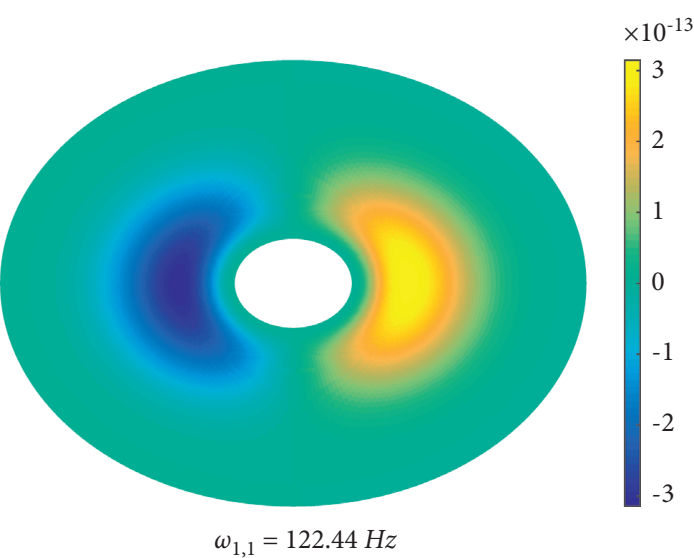

(d) 


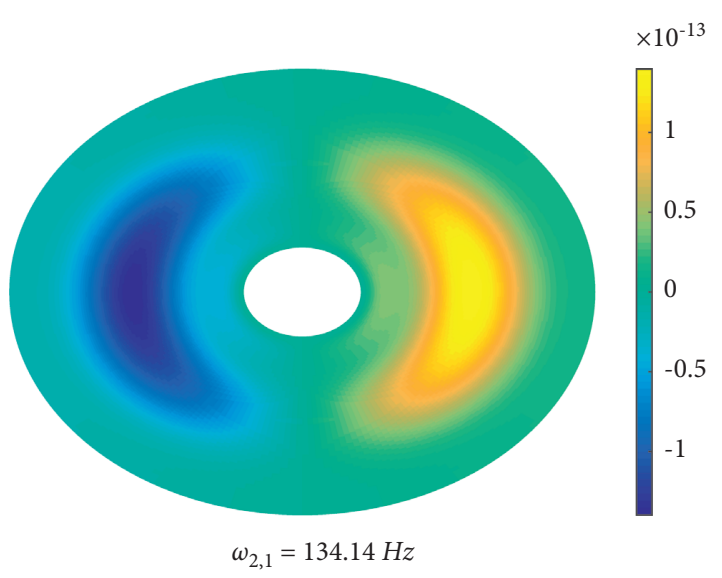

(e)

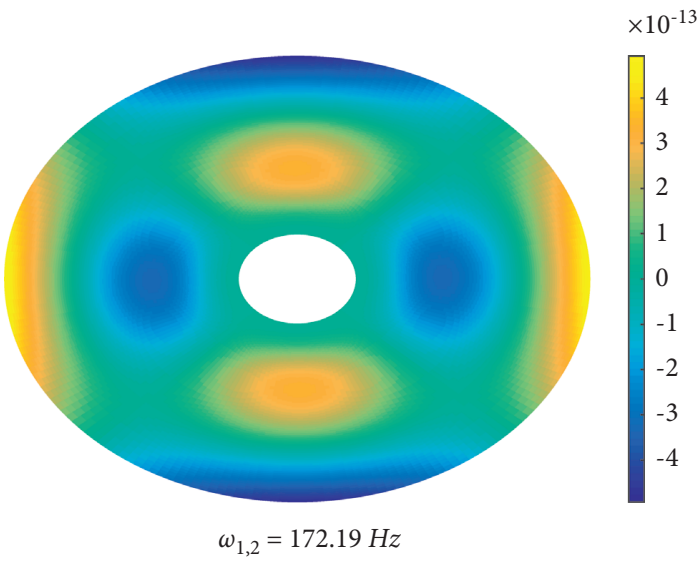

(f)

Figure 4: The mode shapes and the natural frequencies of the rotating disk. (a) $\omega_{0,0}=2.48 \mathrm{~Hz}$. (b) $\omega_{1,0}=31 \cdot 63 \mathrm{~Hz}$. (c) $\omega_{0,1}=74 \cdot 16 \mathrm{~Hz}$. (d) $\omega_{1,1}=122.44 \mathrm{~Hz}$. (e) $\omega_{2,1}=134.14 \mathrm{~Hz}$. (f) $\omega_{1,2}=172.19 \mathrm{~Hz}$.

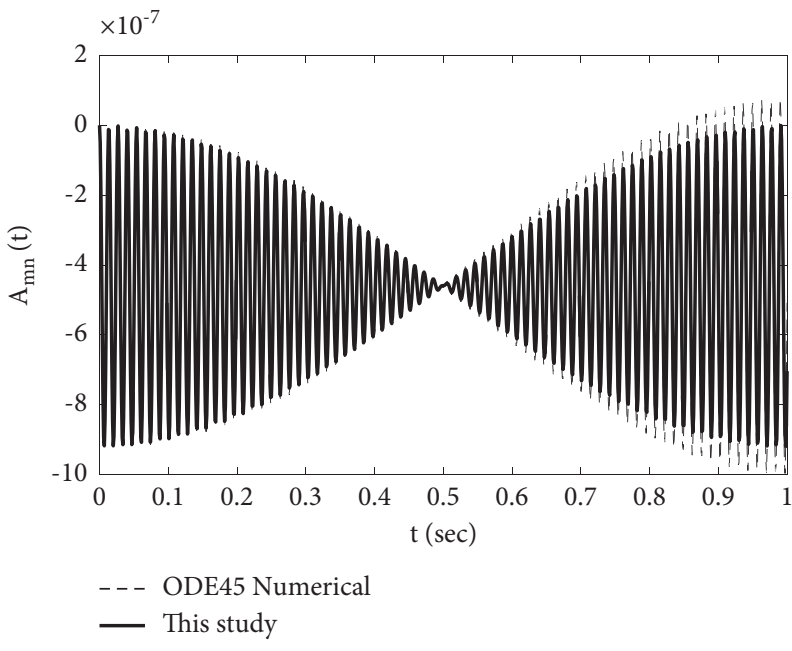

(a)

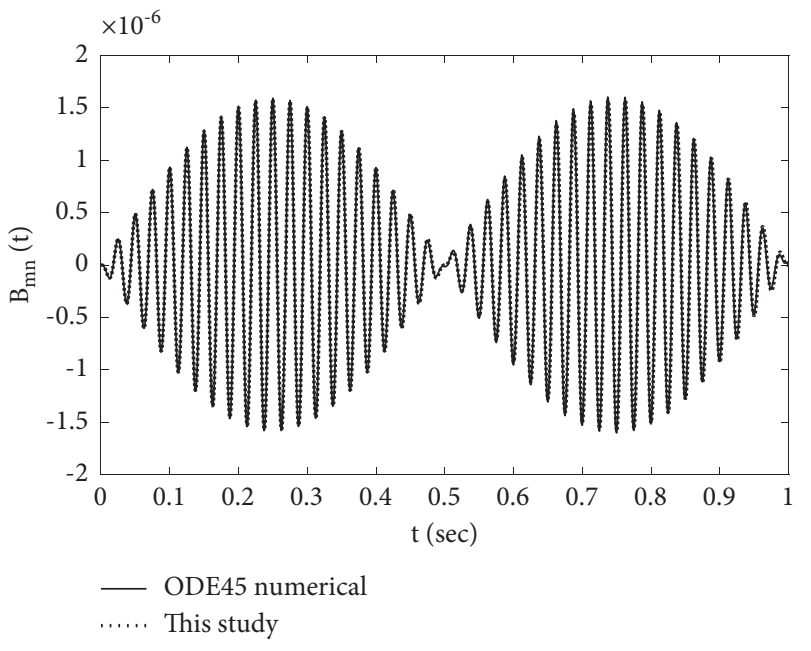

(b)

Figure 5: The time-dependent coefficients of (a) $A_{m n}(\mathrm{t})$ and (b) $B_{m n}(\mathrm{t})$ using analytical and numerical methods.

see a coincidence between two results at the beginning. This low quality of the TMD at the initial times is because the respite TMD needs to be adapted in the system.

The acoustic pressure radiated from the disk was plotted against the time in Figure 11. This figure compares the analytical model of present study with numerical models. A good agreement between the results is observed specially after the transient response.

Figure 12 focuses on the effect of annulus radiuses on the acoustic pressure. It is clear from the figure that increasing the ratio of outer to inner radius of the disk (i.e., b/a) causes dominant increasing in sound pressure. Figures 13(a) and 13(b) describe the effect of the rotational speed on the acoustic response. In Figure 13(a), this effect has been illustrated for different external forces while Figure 13(b) shows these evolutions when the thickness of the disk changes. Form Figures 13(a) and 13(b), one can find a certain frequency (about $800 \mathrm{~Hz}$ in our study) in which the sound radiated from the spinning disk generates its minimum pressure. This frequency is independent of any changes in the force amplitude and the disk thickness.

Figures 14(a) through 14(c) demonstrate time snapshots of the acoustic pressure distribution at the plane parallel to the surface of the rotating disk. The figures were generated when the disk has been rotated a half cycle, one, and oneand-a-half cycles, respectively. It can be found from the figure that the acoustic pressure has the minimum and the maximum values in certain points around the disk, but the field is completely symmetric. Comparing Figures 14(a) through 14(c), one can see the changes in the sound pressure intensity and the distribution pattern. It is also clear from Figures 14(a) and 14(c) that the sound pressure will be amplified in the higher cycles of rotation, but the distribution pattern will be periodic. 


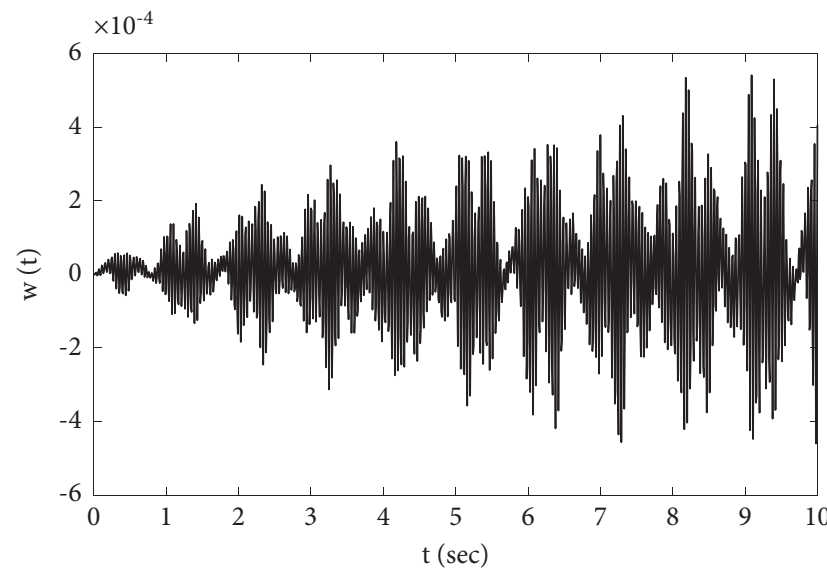

(a)

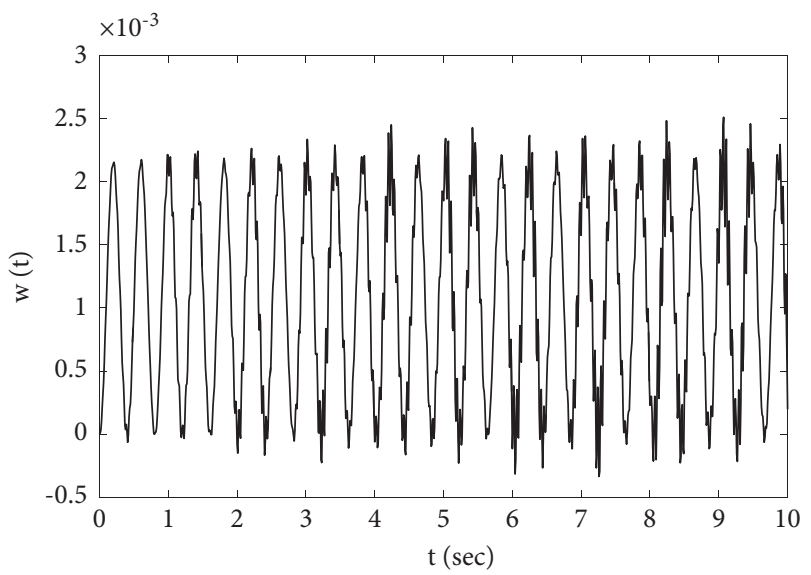

(c)

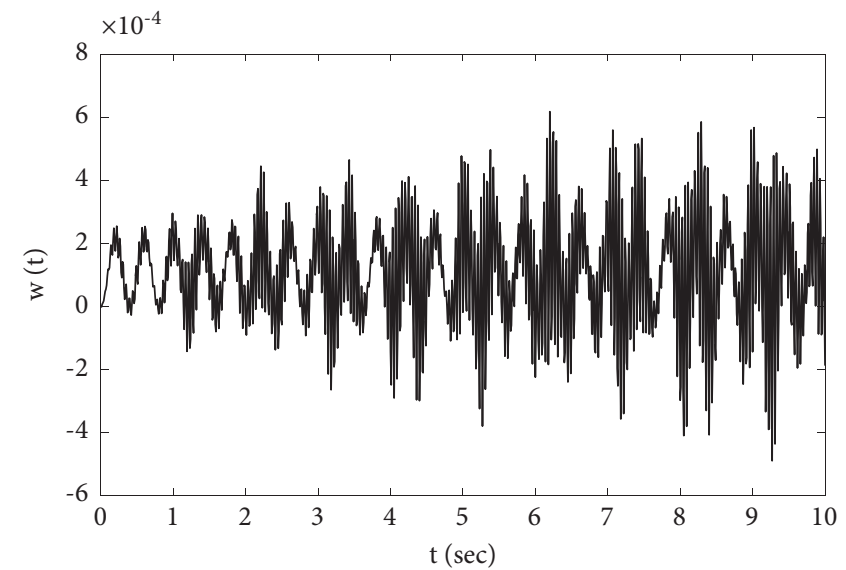

(b)

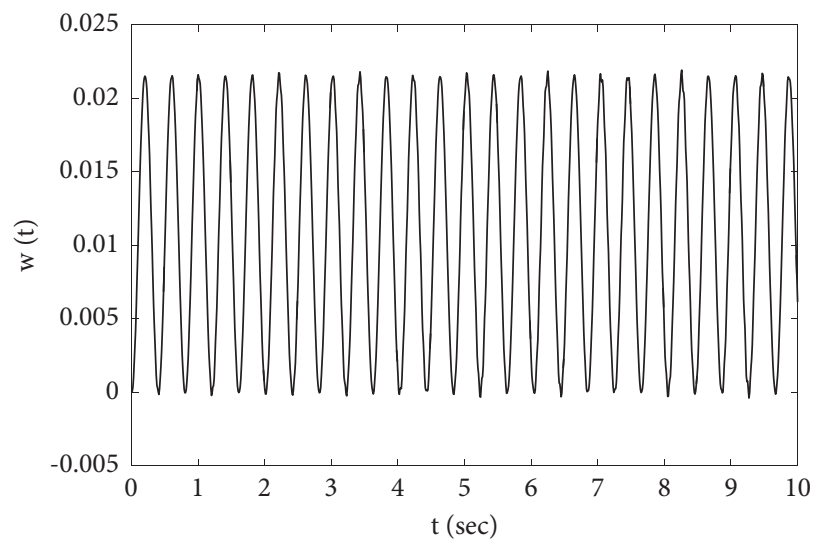

(d)

Figure 6: Time response of the disk with the rotational speed of $\Omega=5 \pi \mathrm{rad} / \mathrm{s}$ for (a) $F=0.1$, (b) $F=1$, (c) $F=10$, and (d) $F=100$.

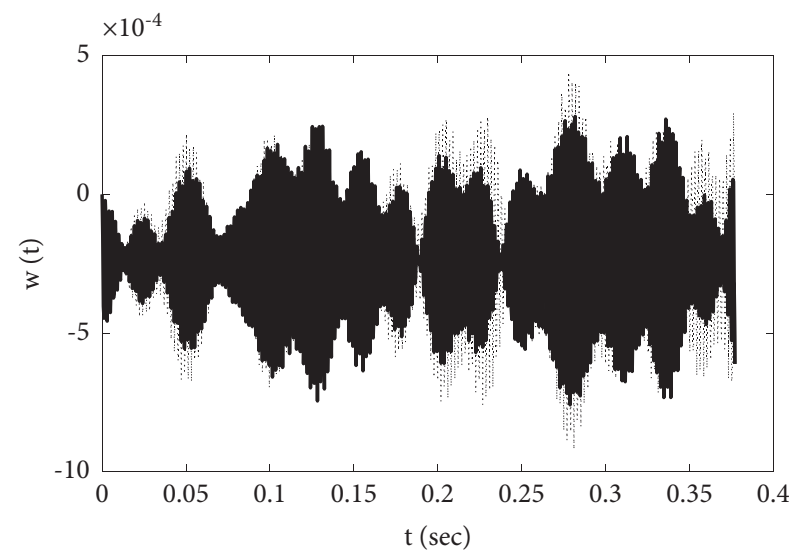

..... non-absorbed deflection

— absorbed deflection

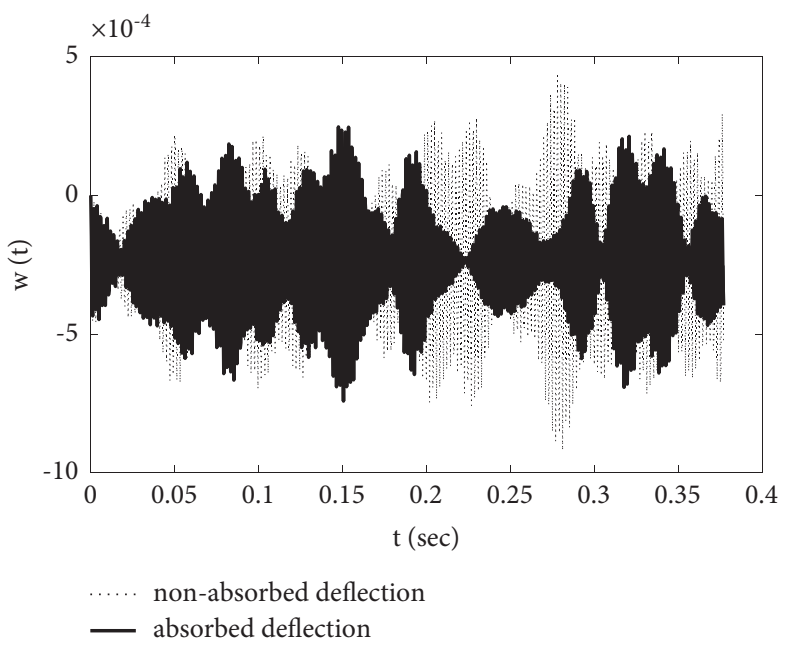

(b)

Figure 7: Continued. 


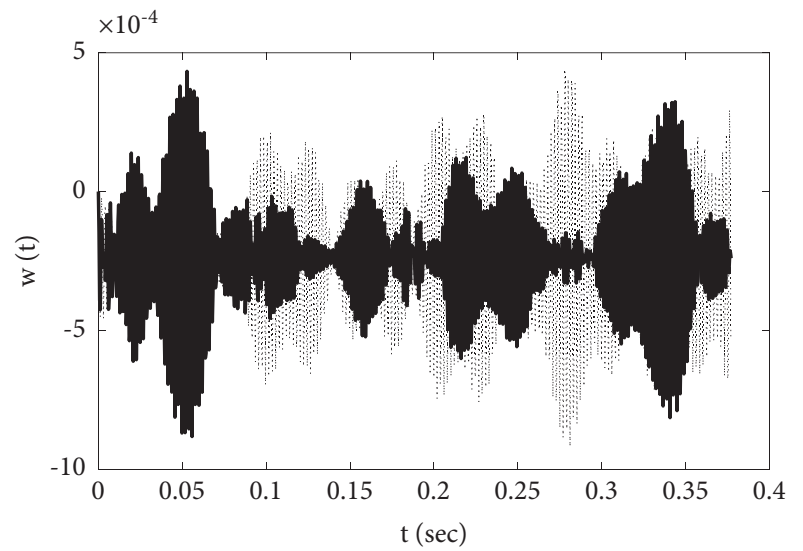

non-absorbed deflection — absorbed deflection

(c)

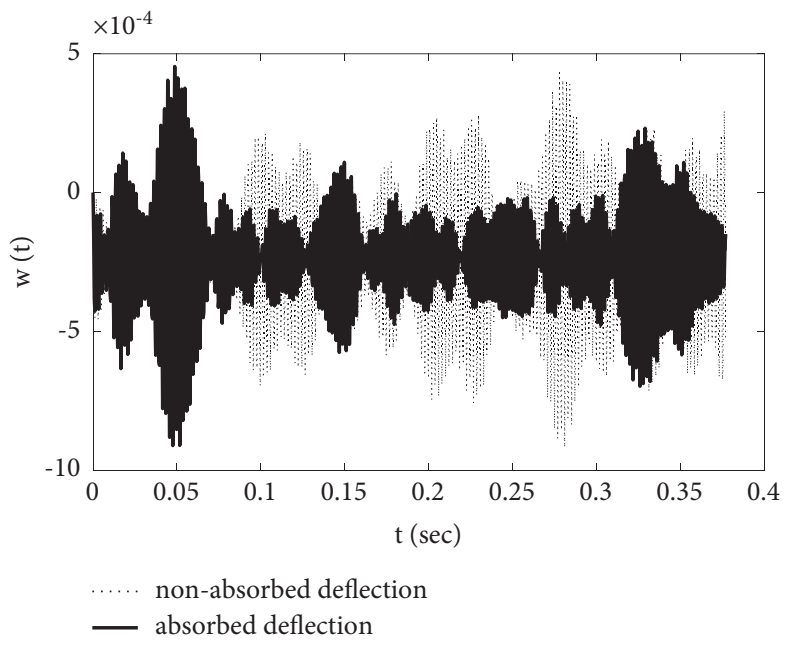

(d)

Figure 7: Time response of the disk with and without TMD for (a) $\omega_{a}=1000 \mathrm{~Hz}$, (b) $\omega_{a}=2000 \mathrm{~Hz}$, (c) $\omega_{a}=3000 \mathrm{~Hz}$, and (d) $\omega_{a}=4000 \mathrm{~Hz}$.

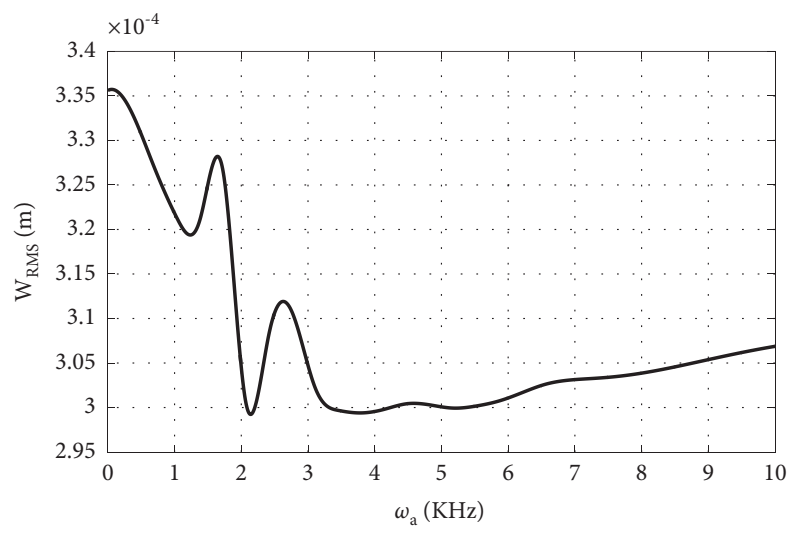

FIGURE 8: RMS of the vibrational response of the disk versus the natural frequency of the dynamic absorber.

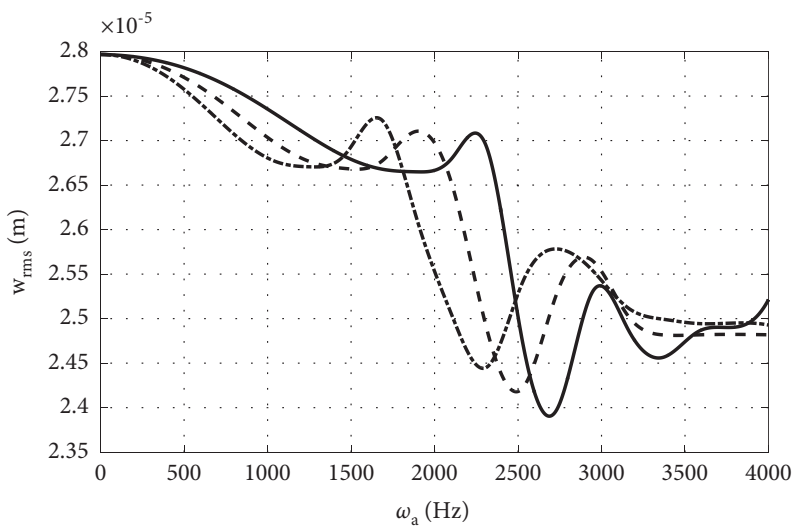

$$
\begin{array}{ll}
-r_{a}=407.5 \mathrm{~mm} \\
---r_{a}=425 \mathrm{~mm} \\
---r_{a}=442.5 \mathrm{~mm}
\end{array}
$$

FIGURE 9: RMS of the transverse response versus the natural frequency of the dynamic absorber for different locations where the TMD attached on. 


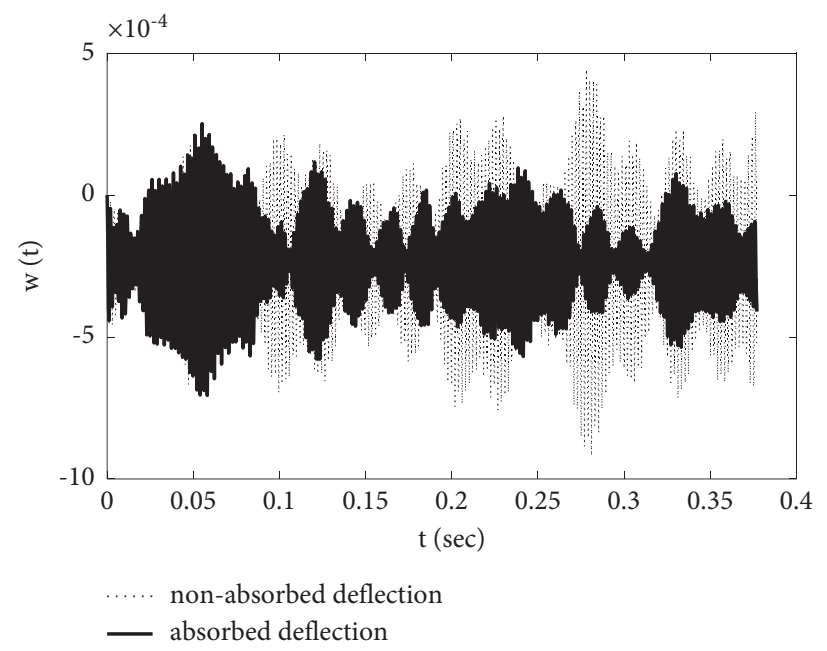

Figure 10: The transverse response evolutions versus time for the dynamic absorber with optimum performance.

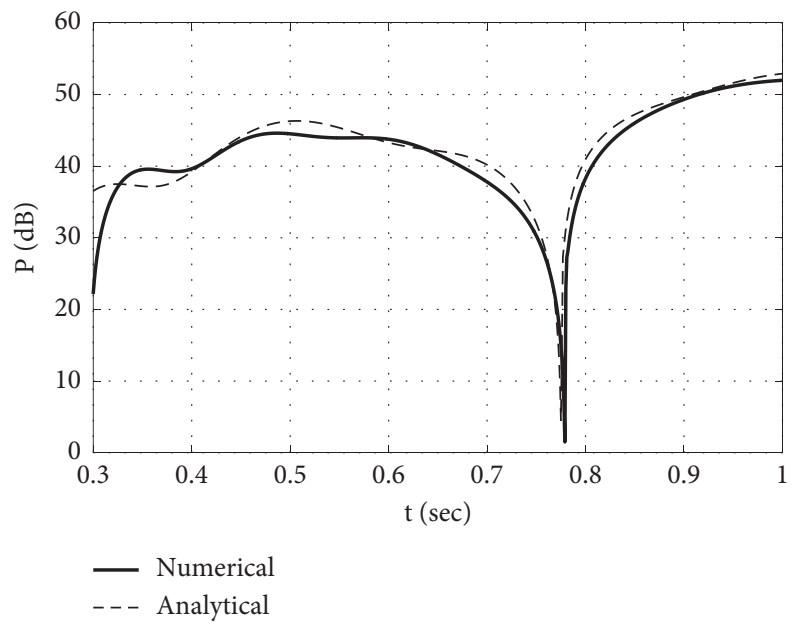

Figure 11: The acoustic pressure radiated from the disk versus time for analytical and numerical modeling.

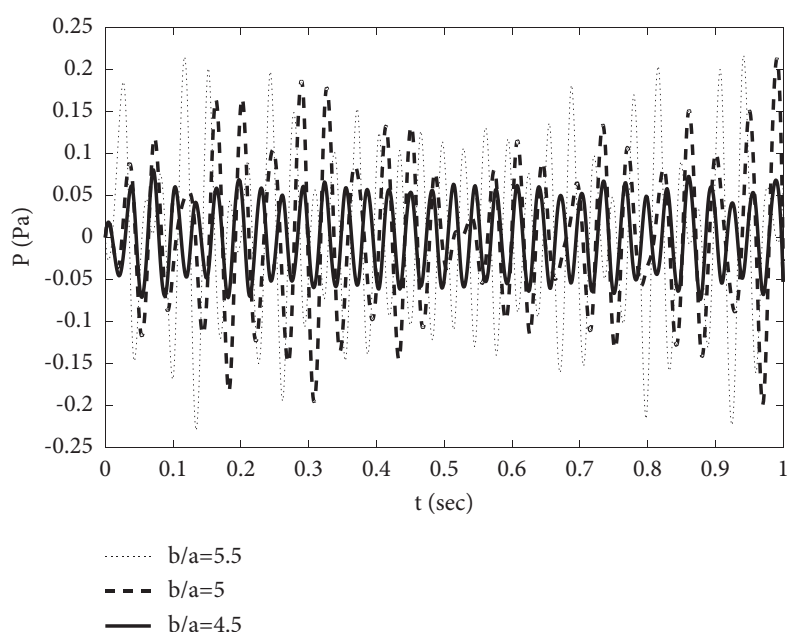

Figure 12: The effect of the ratio of outer to inner radius of the disk on the acoustic pressure. 


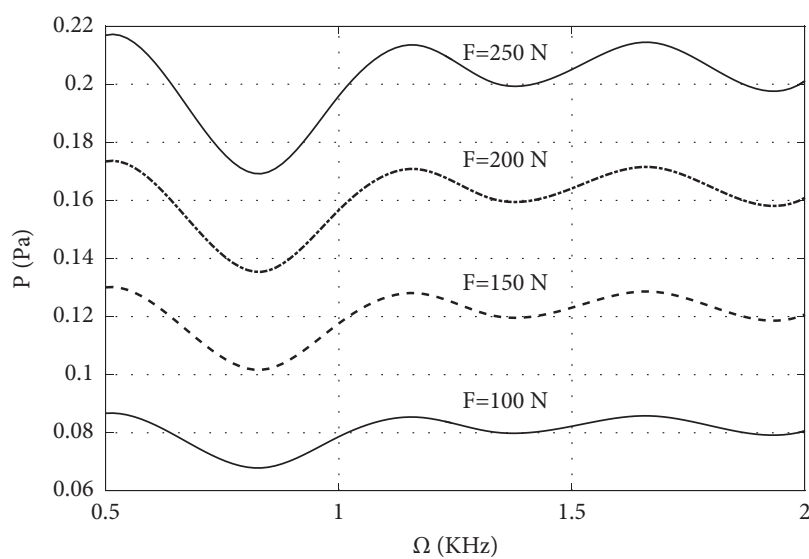

(a)

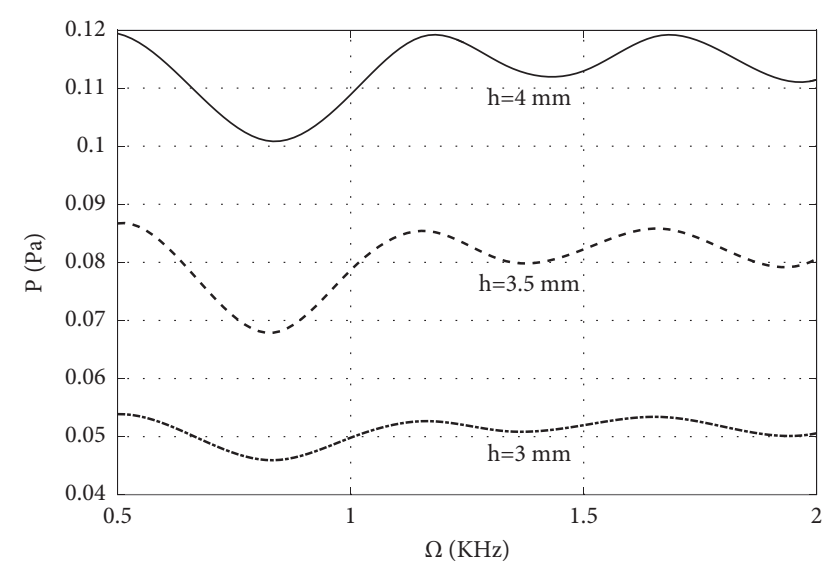

(b)

Figure 13: The acoustic pressure evolutions against the rotational speed of the disk for different (a) external force amplitudes and (b) thicknesses.

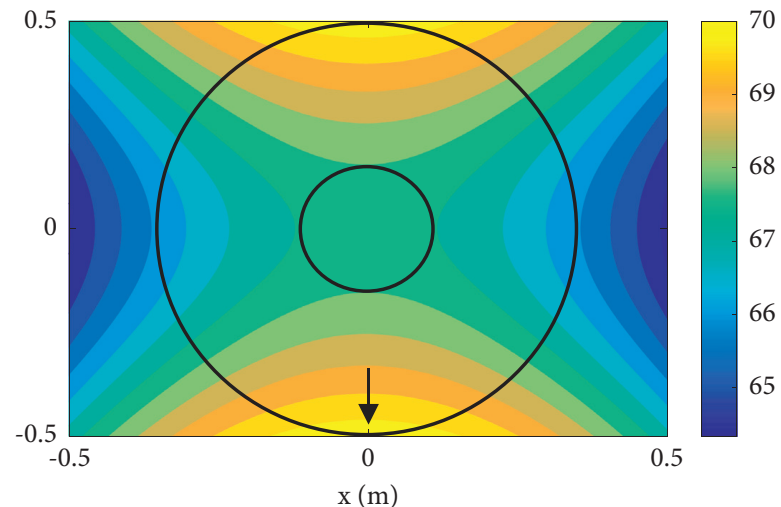

(a)

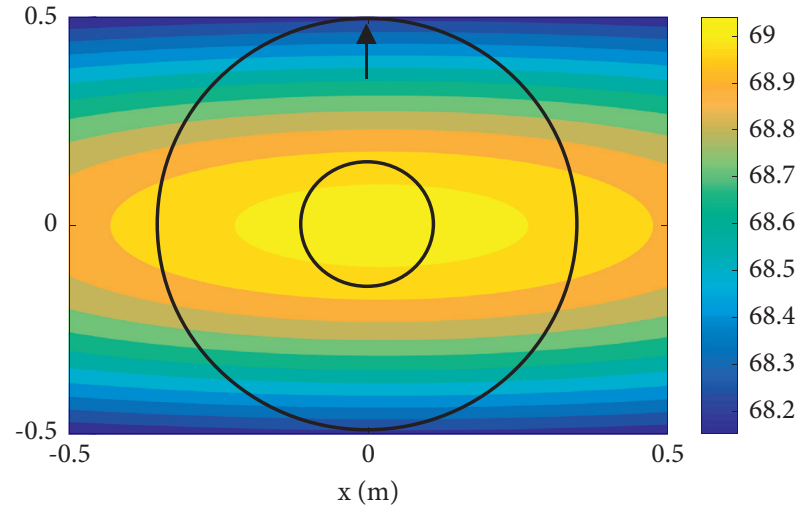

(b)

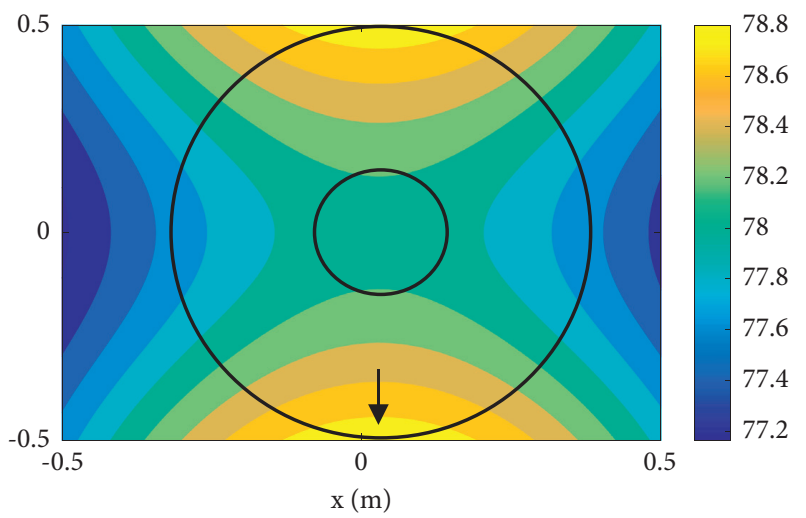

(c)

Figure 14: The acoustic pressure field (dB) parallel to the disk when the disk has been rotated (a) a half cycle, (b) exactly one cycle, and (c) one-and-a-half cycle.

The time snapshots of the acoustic pressure field at the plane parallel to the surface of the system of dynamic absorber-rotating disk showing the location of the TMD have been illustrated in Figures 15(a) through 15(c). Comparing these figures with Figures 14(a) through 14(c), one can obtain the acoustic pressure decreasing after using TMD on the surface of the rotating disk. Furthermore, the acoustic field at the plane parallel to the surface of the disk is asymmetric when the dynamic absorber is attached. One can observe the reduction in the acoustic pressure radiated from the vibrating disk after using TMD. 


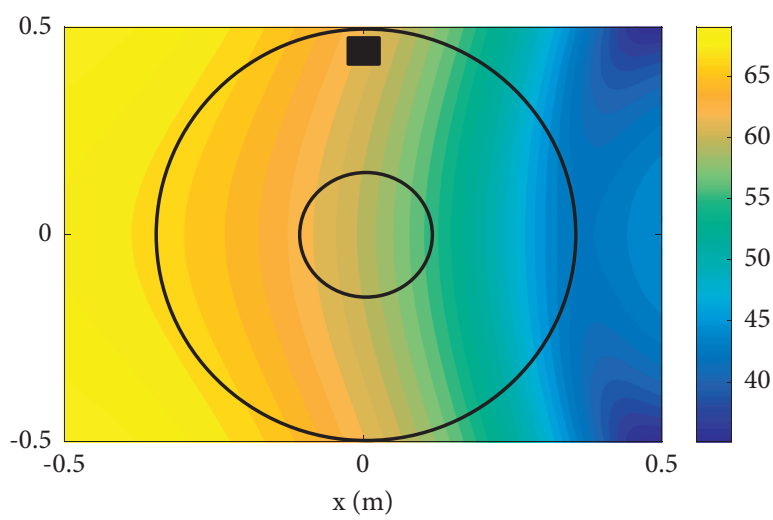

(a)

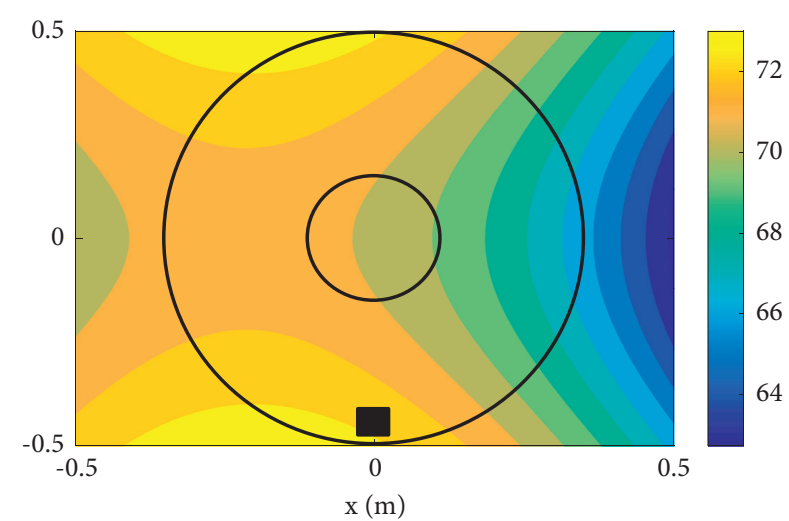

(b)

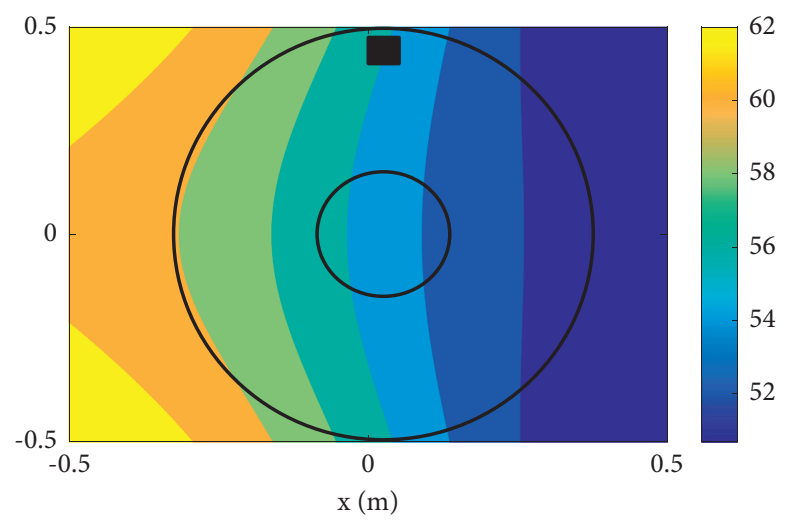

(c)

FiguRE 15: The acoustic pressure field $(\mathrm{dB})$ parallel to the system of dynamic absorber-rotating disk when the disk has been (a) a half cycle, (b) exactly one cycle, and (c) one-and-a-half cycle.

\section{Conclusions}

The analytical modeling of free and forced vibration and the acoustic radiation mitigation for an annular rotating disk was studied in this paper. Galerkin's expansion method was employed to obtain the vibrational response of the disk. The natural frequencies and the mode shapes of the disk were found by analytical methods. Time-dependent coefficients were obtained analytically, and the results were compared with the numerical simulation results. A tuned-massdamped (TMD) mechanism was added to the main system in order to reduce the amplitude of the vibrations. The influence of the different parameters on the efficiency of the TMD was examined. Acoustic pressure radiated from the rotating annulus was computed employing Rayleigh integral method, Laplace transform technique, and Durbin's Laplace transform inversion scheme. The most important results can be mentioned as follows:

(i) The acoustic pressure radiated from the vibrating rotating annular disk is increased by increasing the ratio of outer-inner radius.

(ii) The rotating disk generates different sound levels in different spinning speeds. Moreover, there is a certain speed in which the sound pressure radiated from the disk has a minimum value. This merit rotational speed is independent of forcing conditions.

(iii) Rotating disks with higher thicknesses are noisier than the thin disks.

(iv) The acoustic pressure field generated by the disk is symmetric, but adding TMD to the system makes the sound field asymmetric.

\section{Data Availability}

The data that support the findings of this study are available from the corresponding author, upon reasonable request.

\section{Conflicts of Interest}

The authors declare no conflicts of interest in preparing this article.

\section{References}

[1] D. Younesian and M. H. AleGhafourian, "Vibration of circular hallow disks subjected to rotating transverse Loads," International Journal of Civil Engineering \& Building Materials, vol. 2, no. 4, 2012.

[2] H. Norouzi and D. Younesian, "Forced vibration analysis of spinning disks subjected to transverse loads," International 
Journal of Structural Stability and Dynamics, vol. 15, no. 3, Article ID 1450049, 2015.

[3] J. Qiu, X. Huang, D. Wang, and Y. Xiong, "Experiments on the active control of circular disk vibration," Journal of Low Frequency Noise, Vibration and Active Control, vol. 20, no. 4, pp. 229-237, 2001.

[4] J. W. Heo, J. Chung, and J. M. Park, "Vibration and noise reduction of an optical disk drive by using a vibration absorber," IEEE Transactions on Consumer Electronics, vol. 48, no. 4, pp. 874-878, 2002.

[5] E. Ciğeroğlu and H. N. Özgüven, "Nonlinear vibration analysis of bladed disks with dry friction dampers," Journal of Sound and Vibration, vol. 295, no. 3-5, pp. 1028-1043, 2006.

[6] K.-N. Koo, "Vibration analysis and critical speeds of polar orthotropic annular disks in rotation," Composite Structures, vol. 76, no. 1-2, pp. 67-72, 2006.

[7] S. Bashmal, R. Bhat, and S. Rakheja, "In-plane free vibration of circular annular disks," Journal of Sound and Vibration, vol. 322, no. 1-2, pp. 216-226, 2009.

[8] S. Hashemi, S. Farhadi, and S. Carra, "Free vibration analysis of rotating thick plates," Journal of Sound and Vibration, vol. 323, no. 1-2, pp. 366-384, 2009.

[9] S. Żółkiewski, "Damped vibrations problem of beams fixed on the rotational disk," International Journal of Bifurcation and Chaos, vol. 21, no. 10, pp. 3033-3041, 2011.

[10] D. Younesian, M. H. Aleghafourian, and E. Esmailzadeh, "Vibration analysis of circular annular plates subjected to peripheral rotating transverse loads," Journal of Vibration and Control, vol. 21, no. 7, pp. 1443-1455, 2015.

[11] C.-f. Li, H. X. She, W. Liu, and B. C. Wen, "The influence of shaft's bending on the coupling vibration of a flexible bladerotor system," Mathematical Problems in Engineering, vol. 2017, Article ID 7313956, 2017.

[12] M. H. Jalali and B. Shahriari, "Elastic stress analysis of rotating functionally graded annular disk of variable thickness using finite difference method," Mathematical Problems in Engineering, vol. 2018, Article ID 1871674, 2018.

[13] E. Bagheri and M. Jahangiri, "Analysis of in-plane vibration and critical speeds of the functionally graded rotating disks," International Journal of Applied Mechanics, vol. 11, no. 2, Article ID 1950020, 2019.

[14] P. Lyu, J. Du, Y. Wang, and Z. Liu, "Free in-plane vibration analysis of rotating annular panels with elastic boundary restraints," Journal of Sound and Vibration, vol. 439, pp. 434-456, 2019.

[15] A. Presas, D. Valentin, C. Valero, and M. E. Montagut, "Experimental measurements of the natural frequencies and mode shapes of rotating disk-blades-disk assemblies from the stationary frame," Applied Sciences, vol. 9, no. 18, 2019.

[16] Y. Yang, Z. Wang, and Y. Wang, "Thermoelastic coupling vibration and stability analysis of rotating circular plate in friction clutch," Journal of Low Frequency Noise, Vibration and Active Control, vol. 38, no. 2, pp. 558-573, 2019.

[17] E. Ferhatoglu, S. Zucca, D. Botto, J. Auciello, and L. Arcangeli, "Nonlinear vibration analysis of turbine bladed disks with mid-span dampers," in Turbo Expo: Power for Land, Sea, and AirAmerican Society of Mechanical Engineers (ASME), NY, USA, 2020.

[18] C. Li, P. Li, B. Zhong, and X. Miao, "Large-amplitude vibrations of thin-walled rotating laminated composite cylindrical shell with arbitrary boundary conditions," Thin-Walled Structures, vol. 156, Article ID 106966, 2020.

[19] H. She, C. Li, Q. Tang, and B. Wen, "Nonlinear vibration analysis of a rotating disk-beam system subjected to dry friction," Shock and Vibration, vol. 2020, Article ID 7604174 , 2020.

[20] R. Shakeri and D. Younesian, "Broad-band noise mitigation in vibrating annular plates by dynamic absorbers," International Journal of Structural Stability and Dynamics, vol. 16, no. 6, Article ID 1550014, 2016.

[21] H. Lee and R. Singh, "Acoustic radiation from out-of-plane modes of an annular disk using thin and thick plate theories," Journal of Sound and Vibration, vol. 282, no. 1, pp. 313-339, 2005.

[22] C. C. Cheng, F. T. Wu, and K. L. Ho, "Reduction of flowinduced vibration and noise of an optical disk drive," Journal of Sound and Vibration, vol. 320, no. 1, pp. 43-59, 2009.

[23] M. Maeder, R. D. Auria, E. Grasso et al., "Numerical analysis of sound radiation from rotating discs," Journal of Sound and Vibration, vol. 468, Article ID 115085, 2020.

[24] Z. Wang, Y. Jiao, Z. Chen, X. Qu, and T. Fu, "An analytical study of average radiation efficiency of rotating annular plates in rotating frame," Applied Acoustics, vol. 174, Article ID 107786, 2021.

[25] H. Norouzi and D. Younesian, "Vibro-acoustic numerical analysis for the geometrically nonlinear viscoelastic rectangular plate subjected to subsonic compressible airflow," Applied Acoustics, vol. 174, Article ID 107779, 2021.

[26] D.-S. Liang, H.-J. Wang, and L.-W. Chen, "Vibration and stability of rotating polar orthotropic annular disks subjected to a stationary concentrated transverse load," Journal of Sound and Vibration, vol. 250, no. 5, pp. 795-811, 2002.

[27] M. A. Hajji, Q. M. Al-Mdallal, and F. M. Allan, “An efficient algorithm for solving higher-order fractional Sturm-Liouville eigenvalue problems," Journal of Computational Physics, vol. 272, pp. 550-558, 2014.

[28] F. T. K. Au and M. F. Wang, "Sound radiation from forced vibration of rectangular orthotropic plates under moving loads," Journal of Sound and Vibration, vol. 281, no. 3, pp. 1057-1075, 2005.

[29] F. Durbin, "Numerical inversion of Laplace transforms: an efficient improvement to dubner and abate's method," The Computer Journal, vol. 17, no. 4, pp. 371-376, 1974.

[30] J.-F. Blais and A. Ross, "Forward projection of transient sound pressure fields radiated by impacted plates using numerical Laplace transform," Journal of the Acoustical Society of America, vol. 125, no. 5, pp. 3120-3128, 2009. 